\title{
Oligomeric composition of polyols from fatty acid methyl ester: the effect of ring- opening reactants of epoxide groups
}

\begin{abstract}
ABSTRCT
Commercial availability of fatty acid methyl ester (FAME) from palm oil targeted for biodiesel offers a good feedstock for the production of structurally well-defined polyols for polyurethane applications. The effect of molecular weight (MW), odd and even carbon numbers, and the linear and branched structure reactants used in the ring-opening reaction of epoxidized fatty acid methyl ester (E-FAME) on the properties of polyols was investigated. Conversions of E-FAME to PolyFAME polyols were confirmed by Fourier transform infrared analysis, oxirane oxygen content, and hydroxyl number. Gel permeation chromatography (GPC) calibrated against polyether polyols as a standard and vapor pressure osmometry were used for MW determination. GPC chromatograms of PolyFAME polyols clearly demonstrated the formation of oligomers during ring-opening reactions. MW, and odd and even carbon numbers in a structure of linear diols and branched diol used in the syntheses of PolyFAME polyols did not have an effect on crystallinity, glass transition, or melt temperatures measured using Differential scanning calorimetry (DSC). PolyFAME polyols ring-opened with water, methanol, and 1,2-propanediol contained secondary hydroxyl groups, whereas PolyFAME polyols ring-opened with linear diols contained a mixture of primary and secondary hydroxyl groups. It was found that the concentration of primary hydroxyl groups increased significantly by increasing the number of carbons from C2 to C3 in the linear diols. The viscosity of PolyFAME polyols also increased with the MW of linear diols used in the E-FAME ring-opening reaction. These findings would be beneficial for formulators in choosing the most cost effective polyols for polyurethane formulations.
\end{abstract}

Keyword: Biodiesel; Bio-based polyols; Oligomerization; Molecular weight reactants; Thermal properties. 